\title{
Texture-adaptive image colorization framework
}

\author{
Michal Kawulok and Bogdan Smolka
}

\begin{abstract}
In this paper we present how to exploit the textural information to improve scribble-based image colorization. Although many methods have been already proposed for coloring grayscale images based on a set of color scribbles inserted by a user, very few of them take into account textural properties. We demonstrate that the textural information can be extremely helpful for this purpose and it may greatly simplify the colorization process. First, based on a scribbled image we determine the most discriminative textural features using linear discriminant analysis. This makes it possible to boost the initial scribbles by adjoining the regions having similar textural properties. After that, we determine the color propagation paths and compute chrominance of every pixel in the image. For the propagation process we used two competing path cost metrics which are dynamically selected for every scribble. Using these metrics it is possible to efficiently propagate chrominance both over smooth and rough image regions. Texture-based scribble boosting followed by competitive color propagation is the main contribution of the work reported here. Extensive experimental validation documented in this paper demonstrates that image colorization can be substantially improved using the proposed technique.
\end{abstract}

Keywords: image colorization, textural properties, distance transform, linear discriminant analysis

\section{Introduction}

Color images are usually perceived as definitely more attractive and appealing than their grayscale versions. Therefore, a lot of efforts are often engaged into image colorization, which is a process of adding colors to monochromatic images or videos. First attempts in 1920s were fully manual, performed for every individual shot on the film print. The colorization process was computerized in 1970s by Wilson Markle and Christian Portilla. Its most famous application was colorization of the Apollo mission footage. The first well-known monochrome film colorization was that of Casablanca in 1980s. Although it was widely criticized at that time, colorization of old movies appeared desired in the mass culture world and many films have been converted into color versions since then. Apart from enhancing visual attractiveness of monochrome photographs or videos whose color versions are not available, image colorization has found many other applications like marking regions of interest in medical images, interior design, or make-up simulators.

\footnotetext{
* Correspondence: michal.kawulok@polsl.pl

Faculty of Automatic Control, Electronics and Computer Science, Silesian University of Technology, Akademicka 16, 44-100 Gliwice, Poland
}

Using the recent methods an image can be colorized based on color scribbles which are propagated over the whole image surface. Although the existing techniques work well for colorizing plain areas, they fail for rough, textured regions. This is because the color is propagated from the scribbles following an assumption that pixels of similar luminance should have similar chrominance. This explains why the existing algorithms and available commercial solutions occur to be inefficient when a highly textured regions are to be colorized. In some cases, even large image regions expected to have uniform chrominance should be precisely annotated with the scribbles to avoid artifacts. The final colorization result often depends on the scribbles' shape and exact position. Hence, although the image is automatically colorized after adding the scribbles, drawing them is often a tedious task itself.

In the work reported here we have focused on how to reduce density and precision of the scribbles, in order to simplify the colorization process. More specifically, we have investigated how the textural information can be exploited to achieve this goal. As a result, based on our earlier works $[1,2]$ we propose a double-level method, consisting of scribble boosting followed by surface-specific competitive color propagation. A very important property of the method is that at both levels it is

\section{Springer}


adapted to the textures which appear in the image and are marked by the scribbles.

The first-level works by extracting the discriminative textural features (DTF) which make distinction between the textures covered by different scribbles [1]. DTF are obtained using linear discriminant analysis (LDA) performed over simple image statistics computed locally. After that, the scribbles are boosted by adjoining the regions which have similar textural features. DTF are determined independently for every image to maximize the discriminative power between the textures covered by different scribbles. This makes the method adaptive to every scribbled image.

At the second level, the boosted scribbles serve as the source for the color propagation. The propagation paths are obtained using Dijkstra algorithm by minimizing local pixel distance integrated along the path. In conventional techniques [3] the local pixel distance is proportional to a luminance difference. This works correctly for colorization of plain areas, but fails for textured surface. Therefore, we adapt the distance to the textural properties of the region where the scribble is placed. Our experiments indicated that this double-level approach make it possible to limit the necessary human assistance and facilitates the colorization process.

The paper is organized as follows. In Section 2, a general literature overview is presented. Then, in Section 3, the baseline techniques used in the proposed method are outlined. The main contribution of the reported work is presented in the following two sections. In Section 4, competitive color propagation is described, and in Section 5, we present the texture-based scribble boosting technique. Finally, the obtained colorization results are shown and discussed in Section 6, and the conclusions are presented in Section 7.

\section{Related work}

The first method of adding colors to the image was proposed by Gonzalez and Woods [4] in a form of luminance keying. It operates based on a function which maps every luminance level into color space. Obviously, the whole color space cannot be covered in this way without increasing manual input from the user. Welsh et al. [5] proposed a method of color transfer which colorizes a grayscale image based on a given reference color image. This method matches pixels based on their luminance and standard deviation in $5 \times 5$ neighborhood, which serves as a basic textural feature. Every pixel in the colorized image is assigned the best matching pixel from the source image and its chrominance is transferred. The matching process can be performed automatically, but it gives better results with user assistance. This method was improved by Lipowezky [6], who proposed to extend the textural features.
Sykora et al. [7] proposed an unsupervised method for image colorization by example, which at first matches similar image feature points to predict their color. After that, the color is spread all over the image by probabilistic relaxation. Horiuchi [8] proposed an iterative probabilistic relaxation, in which a user defines colors for selected grayscale values, based on which the image is colorized. Furthermore, Horiuchi [9] proposed a method for texture colorization which defines pixel similarity based on their Euclidean distance and difference in luminance values. Hence, even if two neighboring pixels differ much in luminance, which is often observed for textured regions, their similarity will be high due to low Euclidean distance. This approach works better for colorizing textures than the earlier methods, but it does not perform any analysis of textural features.

Many methods are focused on using prior information delivered by a user in a form of manually added color scribbles. Levin et al. [10] formulated an optimization problem based on an assumption that neighboring pixels of similar intensity should have similar color values under the limitation that the colors indicated in the scribbles remain the same. Yatziv and Sapiro [3] proposed a method for determining propagation paths in the image by minimizing geodesic distances from every scribble. Based on the distances from each scribble, pixel color is obtained by blending scribble chrominances. In other works, the color is also propagated from scribbles with probabilistic distance transform [11], using cellular automaton [12] or by random walks with restart [13].

During our earlier research, we also exploited scribblebased image colorization. First, we proposed modified color propagation paths and we improved the chrominance blending procedure [2]. This method was suitable for colorizing the details having strong gradients, but still required high scribble coverage. Later, we proposed to use textural features as a domain for color propagation [1], which made it possible to colorize larger areas using small scribble coverage. However, the main drawback of that approach lies in the precision. At the boundaries of regions having different texture, the pixels were often misclassified which resulted in observing unnatural artifacts. In the work reported here, we have modified the procedure for obtaining the textural features and proposed the scribble boosting technique, which eliminates the main drawbacks of these earlier algorithms.

\section{Color propagation paths and chrominance blending}

In order to colorize a monochromatic image $Y$ based on a set of $n$ initial scribbles $\left\{S_{i}\right\}, i=1, \ldots, n$, frst it is necessary to determine the propagation paths from each scribble to every pixel in the image. A path from a pixel $x$ to another pixel $y$ is defined as a discrete function $p$ 
$(t):[0,1] \rightarrow Z^{2}$, which maps a position $t$ in the path to the pixel coordinate. The position is an integer ranging from 0 for the path beginning $(p(0)=x)$ to $l$ for its end $(p(l)=y)$. Also, if $p(i)=a$ and $p(i+1)=b$, then $a$ and $b$ are neighboring pixels. The paths should be determined, so as to minimize a number of expected chrominance changes along the path. Hence, in the image they should follow the objects having uniform chrominance. Also, any two pixels inside a region that is supposed to have uniform chrominance are expected to be connected with a path which should not leave this region.

\subsection{Propagation paths optimization}

The propagation paths from a scribble to every pixel are determined by minimizing a total path cost:

$$
C(p)=\sum_{i=0}^{l-1} \rho\{p(i), p(i+1)\}
$$

where $\rho$ is a local dissimilarity measure between two neighboring pixels and $l$ is the path length. The minimization is performed using Dijkstra algorithm [14] in the following way:

1. A priority queue $Q$ is initialized with all scribbled pixels.

2. Distance array $D$ which covers all image pixels is created. Every pixel $q \in Q$ is assigned a zero distance $(D(q \in Q)=0)$ and all remaining pixels are initialized with an infinite distance.

3. A pixel $q$, for which the distance $D(q)$ is minimal in $Q$, is popped from $Q$ and for each of its 7 neighbors $N_{i}(q)$ (excluding the source) two actions are performed:

(a) Local distance $\rho(q, s)$ between $q$ and its neighbor $s$ is calculated to find a total cost of $p_{s}$, i.e., $C\left(p_{s}\right)=C(q)+\rho(q, s)$.

(b) If $C\left(p_{s}\right)<D(s)$, the distance $D(s)$ is updated, $s$ is enqueued in $Q$, and the pixel $s$ is associated with a new path $p_{s}$.

4. If the queue is empty, the algorithm terminates. Otherwise, step (3) is repeated.

The path route depends mainly on how the local costs are computed. Following the conventional approach [3], the local cost is obtained by projecting the luminance gradient onto a line, tangent to the path direction. This means that the cost is proportional to the difference in luminance between the neighboring pixels.

\subsection{Chrominance blending}

Chrominance of each pixel is determined based on the propagation paths from every scribble. Its value is computed as a weighted mean of scribbles' colors with the weights obtained as a function of the total path cost. Usually two or three strongest components are taken into account, which provides a good visual effect of smooth color transitions. The final color value $v(x)$ of a pixel $x$ is obtained as

$$
v(x)=Y(x) \frac{\sum_{i} v_{i} w_{i}(x)}{\sum_{i} w_{i}(x)}
$$

where $v_{i}$ is the chrominance of an $i$ th scribble and $w_{i}$ $(x)$ is its weight in pixel $x$. We use $Y C_{r} C_{b}$ color space and calculate color values separately for $C_{r}$ and $C_{b}$ channels. The weights are obtained as

$$
w_{i}(x)=\left(C_{i}(x)+1\right)^{-2},
$$

where $C_{i}(x)$ is the total path cost from $i$ th scribble to pixel $x$. In our earlier work [2], we justify that it is beneficial to use modified cost $C_{i}^{b}(x)$ for the blending instead of the original path cost, computed as

$$
C_{i}^{b}(x)=\frac{l}{\sigma_{i}} C_{i}(x)+\alpha l,
$$

where $\sigma_{i}$ is $i$ th scribble strength normalized from 0 to $1, \alpha$ is a topological penalty, and $\rho$ indicates the original path cost. By default the topological penalty was set to $\alpha=0.02$ and the scribble strength $\sigma_{i}=1$; this parameter gives the user possibility to indicate how far the scribble is supposed to propagate. This is particularly important when an image is intended to be colorized using few scribbles. In such a case the scribble strength should be decreased for the scribbles which indicate tiny details and therefore should not propagate much.

\section{Competitive propagation paths}

Yatziv [3] in his method determines the path by minimizing integrated luminance gradient in the path direction. This is an interesting approach, appropriate to determine paths supposed to cross easily plain areas without strong edges. It is suitable if luminance difference is proportional to probability of chrominance change. This approach is similar to a traveler who intends to cross an island with beaches along the coast and mountains in its interior part. He would choose a longer way along the coast rather than a shorter one across the mountains. However, if he wants to move between two mountains, he may prefer to head for the coast, follow the beach to get as close the second mountain as possible, and then walk inside again. This is reasonable, but for the colorization purposes we would prefer not to leave the rough area as long as it is expected to have uniform chrominance. Here, the roughness would mean a texture with many edges which would generate a very high cost of crossing it 
using the conventional methods. In practice, this means that the scribbles would not propagate well in such a region, and as a result it must be annotated with many scribbles.

When a scribble is placed in rough area, it is better to follow high gradients without much cost. It is similar to the intelligent scissors [15] for interactive image segmentation. This algorithm joins a starting point and a mouse pointer with a path, which is sticky to the strongest gradient. Local cost between two neighboring pixels depends on the Laplacian zero-crossings, gradient magnitude and direction. Basically, the cost is lower if the path follows the gradient direction and the gradient magnitude of the path pixels is high.

\subsection{Local distance metrics}

Following the presented analysis, we identified two ways of calculating the local distances which are individually appropriate for homogenous and highly textured regions. We called them respectively: plain distance and gradient-sticky distance.

Plain distance is similar to those used in other wellestablished methods. Its aim is to minimize intensity changes along the path and it is calculated as:

$$
\rho_{p}(x, y)=1-\exp \left(-|Y(x)-Y(y)| / h_{p}\right),
$$

where $h_{p}$ is a normalization factor, set experimentally to 30. This distance is suitable for determining paths in uniform regions whose texture is not characterized by strong gradients.

However, for objects whose texture is not smooth, the paths cannot be found correctly in this way.
Furthermore, the distance grows rapidly when high gradients are crossed, which affects the result of chrominance blending. Therefore, in such cases the distance should be inversely proportional to the gradient strength, so that the path is sticky to high gradients. Hence, we take into account the propagation direction to decrease the cost if the path follows an edge. We defne a gradient-sticky distance as:

$$
\rho_{g}(x, y)=1-\exp \left[\frac{-1}{h_{g}|\nabla Y(\gamma)|(\cos \beta+1)}\right],
$$

where $\beta$ is an angle between the gradient vector in $y$ and propagation direction from $x$ to $y$. Factor $h_{g}$ was set to 0.5 .

Propagation paths obtained by minimizing these two distances integrated along the path, as well as the conventional distance metric defined by Yatziv [3], are presented in Figures 1 and 2. Figure 1 shows the paths propagated from scribbles placed over highly textured regions (hair and tree). In the background, a gradient magnitude image is presented for the upper row and original image for the tree in the bottom row. It may be noticed that in (c) the paths are sticky to the gradient directions, while in (a, b) they prefer smooth areas. Moreover, in the bottom row $(a, b)$ the left part of the tree is accessed by the paths which first leave the tree region, go round the tree through the sky region, and enter the tree region again from the opposite side. This is a good illustration of the traveler's problem described at the beginning of this section. As a result, the left region of the tree would be influenced by scribbles annotated over the sky. a)
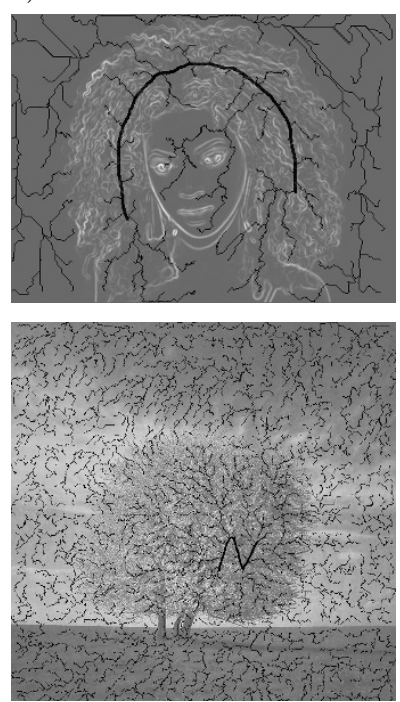

b)
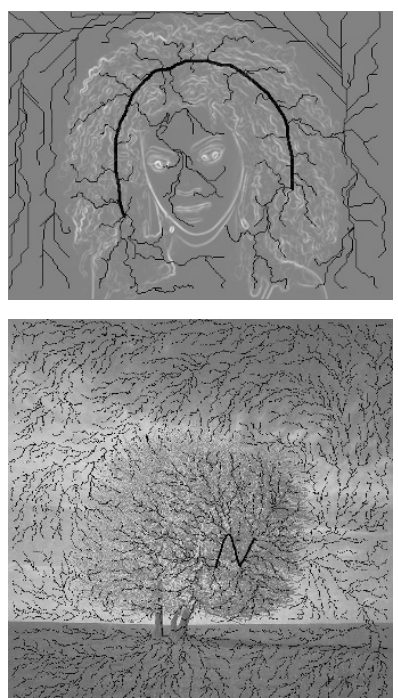

c)
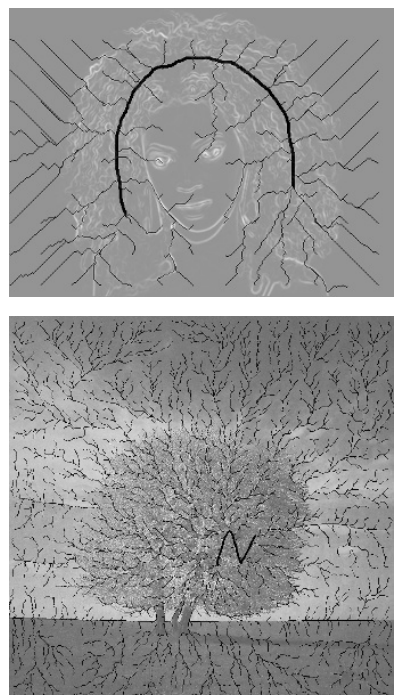

Figure 1 Propagation paths determined using distance defined by Yatziv [3](a), plain distance (b), and gradient-sticky distance (c) 
a)

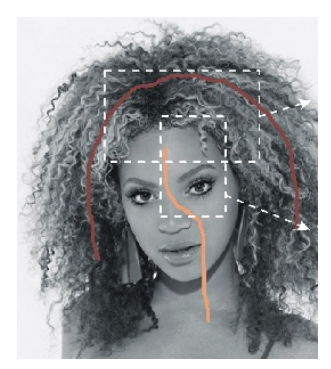

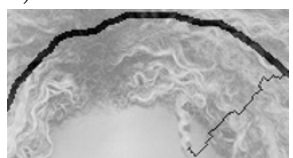

$C(p)=0.35$

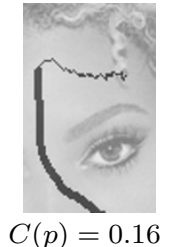

b)

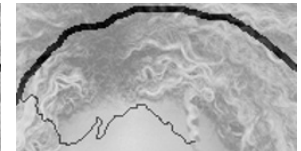

$C(p)=4.6$

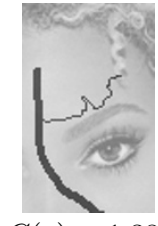

$C(p)=1.38$ c)

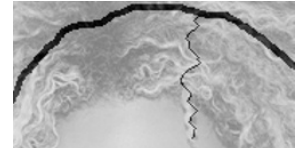

$C(p)=1.14$

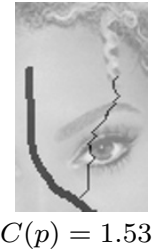

Figure 2 A single point reached by paths obtained with distance defined by Yatziv [3](a), plain distance (b), and gradient-sticky distance (c).

Figure 2 presents propagation paths to a selected pixel of a human hair, reached from two different scribbles added to hair and skin region. The total path cost is depicted in this figure. The path leading from the hair scribble should not leave the hair region which is obtained only using the gradient-sticky distance (c). However, the path leading from the skin scribble is correct only for plain distances $(a, b)$. In case of the gradient-sticky distance, the path crosses an eye which is definitely incorrect.

This example clearly shows that the distance type used for determining a path should depend on the properties of a texture which is to be colorized. This choice may be left to a user who adds the scribbles. However, in our method we intend to decrease the time-consuming interaction, so we provide automatic selection following a competitive approach. For every scribble we start the propagation algorithm with both types of paths and for each pixel we select that kind of a path, for which the distance is smaller. Hence, for harsh surfaces the gradient paths usually prevail, while on smooth areas the plain paths propagate better. This selection can be done either separately for every starting pixel or for a whole scribble. In our experiments, we found the latter approach performing better.

Competitive propagation can be effective only if the competing metrics are well balanced. Otherwise, one would dominate the other. Exponential distance definition in (5) and (6) normalizes plain and gradient-sticky distances. A proper balance between them is achieved using appropriate values of the normalization factors $h_{g}$ and $h_{p}$. It is worth observing that the propagation is performed for small values of the local distances, where the dependence is close to linear.

\section{Texture-based image colorization}

Competitive propagation paths presented in Section 4 allow for efficient colorization despite of strong gradients that are often observed in textured regions. This makes it possible to colorize such image areas using just a few scribbles, similarly as in case of smooth regions. However, this technique does not extract the underlying textural features, so the propagation paths can easily cross boundaries between different textures. It is worth noting that regions of uniform texture quite often have similar chrominance, and chrominance boundaries may be determined based on the textural features. Unfortunately, this is neglected by many existing techniques, which assume that the chrominance boundaries are correlated exclusively with the luminance changes. Following this assumption, the raw pixel values in luminance channel are used as the color propagation domain $[3,16]$.

In this section, we focus on how to exploit the textural features for image colorization. At first, we determine which textural features are most discriminating between the scribbles to obtain appropriate color propagation domain, adapted to the specific conditions. Subsequently, we allow the scribbles to conquer the regions of similar texture, without defining the exact color boundaries (the precision at the boundaries is unsatisfactory). After this procedure, which we call scribble boosting, we perform the competitive propagation as described earlier in this paper.

\subsection{Discriminative textural features}

Various methods have been reported on texture-based image segmentation [17], including Haralick features [18], local binary patterns [19], wavelets [20], or filter banks [21]. It is worth noting that the considered case is not identical to the widely investigated segmentation task. Here, the aim is to define a suitable domain for color propagation. Among the existing colorization methods, textural features have been exploited for color transfer [5,6]. However, only simple texture descriptors are used there, which may be helpful in some cases, but 
does not guarantee the distinctiveness between the regions marked with different scribbles.

The color propagation domain should induce low costs between pixels belonging to a single scribble. On the other hand, the cost should be high, when the path crosses a boundary between areas marked with different scribbles. It is therefore important to find such image properties that would be uniform within a single scribble and different between the scribbles. In the work reported here, we select the distinctive properties for every scribbled image using LDA. It is performed over a set of simple image features extracted from pixels which belong to the scribbles. In this way we obtain the color propagation domain which is dynamically conformed to every specific case.

\subsubsection{Linear discriminant analysis}

Linear discriminant analysis [22] is a supervised statistical feature extraction method frequently used in machine learning. It finds a subspace defined by the most discriminative directions within a given training set of $M$-dimensional vectors classified into $K$ classes. The analysis is performed first by computing two covariance matrices: within-class scatter matrix $S_{W}=\sum_{i=1}^{K} \sum_{u_{k} \in K_{i}}\left(u_{k}-\mu_{i}\right)\left(u_{k}-\mu_{i}\right)^{T}$, and betweenclass scatter matrix $S_{B}=\sum_{i=1}^{K}\left(\mu_{i}-\mu\right)\left(\mu_{i}-\mu\right)^{T}$, where $\mu$ is a mean vector of the training set and $\mu_{i}$ is a mean vector of the $i$ th class (termed $K_{i}$ ). Subsequently, the matrix $S=S_{W}^{-1} S_{B}$ is subjected to the eigen decomposition $S=\Phi \Lambda \Phi^{T}$, where $\Lambda=\operatorname{diag}\left(\lambda_{1}, \ldots, \lambda_{M}\right)$ is the matrix with the ordered eigenvalues along the diagonal and $\Phi$ $=\left[u_{1}|\ldots| u_{M}\right]$ is the matrix with the correspondingly ordered eigenvectors as columns. The eigenvectors form the orthogonal basis of the feature space. Originally, the feature space has $M$ dimensions, but only those associated with the highest eigenvalues have strong discriminative power, while the remaining can be rejected. In this way the dimensionality is reduced from $M$ to $m$, where $m<M$.

After having built the $m$-dimensional feature space, the feature vectors are obtained by projecting the original vectors $u$ onto the feature space: $v=\Phi^{T} u$. The similarity between the feature vectors is computed based on their Euclidean distance in the feature space.

\subsubsection{LDA for texture analysis}

In order to determine the discriminative features, first we calculate basic image features from every pixel. They are composed of: (a) luminance, (b) gradient intensity, (c) local binary pattern, (d) mean value and (e) standard deviation computed in many kernels of different size, (f) the difference between maximum and minimum values in the kernels, and (g) the pixel value in the median filtered image. The basic features (d)-(g) were obtained for five kernel sizes ranging from $3 \times 3$ to $11 \times 11$. Hence, every pixel $x$ is described by an $M$-dimensional basic feature vector $u_{x}(M=23$ in the presented case). The feature vectors of the scribble pixels are subsequently subject to LDA. Every scribble forms a separate class, so the analysis determines the most discriminative features between the scribbles for a given image. The feature vectors $(v)$ obtained using LDA are further termed discriminative textural features (DTF). The distance between any two feature vectors $v_{1}$ and $v_{2}$ in the DTF space is computed as:

$$
d_{\mathrm{DTF}}=\sum_{i=1}^{m}\left(v_{1 i}-v_{2 i}\right)^{2} .
$$

During our experiments, we observed that for the majority of analyzed cases it is sufficient to reduce the dimensionality of DTF vectors to $m=2$. Also, we limit the number of the input vectors in each class to 100 so as to reduce the LDA training time. If a scribble contains more pixels, 100 of them are randomly selected. We have not observed any noticeable difference in the outcome compared to using all the scribble pixels, while the training time is definitely shorter.

\subsection{DTF-based color propagation domain}

After training, a projection matrix $\Phi$ is obtained and every pixel in the image is projected onto $m$-dimensional DTF space. Examples of three scribbled images and their projection onto three leading LDA components are shown in Figure 3. They represent the most discriminative textural features and the eigenvalues associated with them are given underneath. It may be observed that these projections differentiate well between the areas marked with the scribbles. Also, 10 highest eigenvalues obtained for every image are plotted in the figure (rightmost column). The values on the vertical axis are given in relation to the highest eigenvalue.

Figure 4 shows four images annotated with scribbles. The luminance of the pixels scaled from 0 to 100 is shown in (b) on the horizontal axis, while the vertical axis was added only to differentiate between the scribbles. Different colors (red, blue, and green) indicate pixels from particular scribbles. The scribble pixels projected onto 2D DTF subspace are shown in (c). For the image in the first row, the "forest" pixels (F-blue) are generally darker than the "sky" pixels (S-red), but the luminance alone is not a discriminative feature here. However, two classes are well separated after projecting onto the DTF subspace, and the same observation concerns the flower image. Two subsequent images were annotated with scribbles of three various colors, each of them being a separate class. "Sky" (S-green) and "grass" 


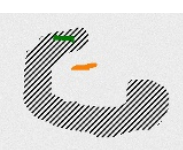

a)

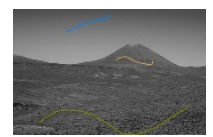

b)

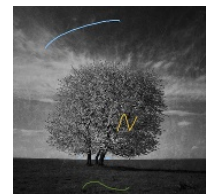

c)

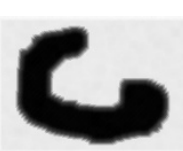

$\lambda_{1}=1361$

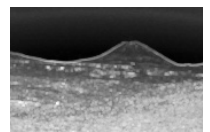

$\lambda_{1}=758$

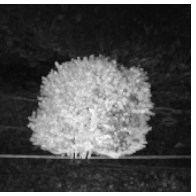

$\lambda_{1}=965$

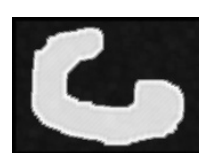

$\lambda_{2}=946$

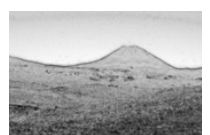

$\lambda_{2}=569$

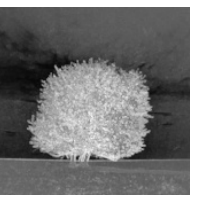

$\lambda_{2}=727$

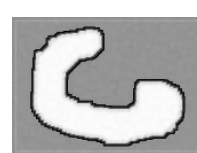

$\lambda_{3}=263$

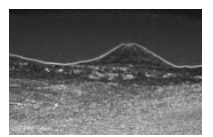

$\lambda_{3}=335$

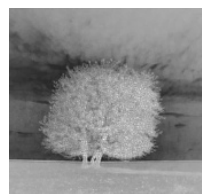

$\lambda_{3}=488$

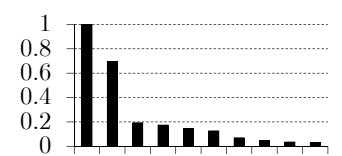

$\begin{array}{lllllllll}12 & 3 & 4 & 5 & 6 & 7 & 8 & 9 & 10\end{array}$ Eigenvalue

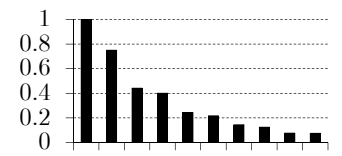

1233456788910

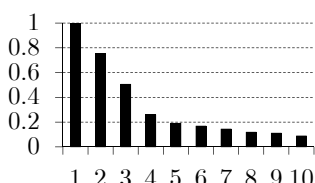

Eigenvalue

Figure 3 Projections of scribbled images onto the leading LDA components, and 10 highest eigenvalues

a)
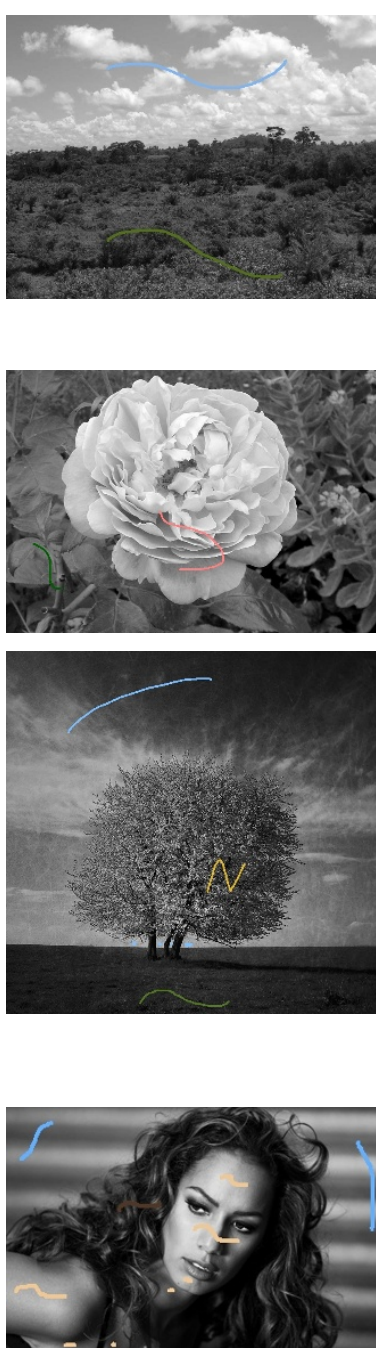

b)

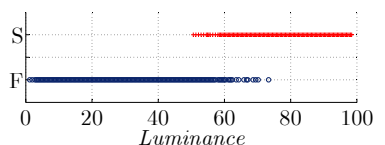

S - sky scribble

$\mathrm{F}$ - forest scribble

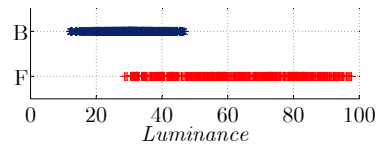

B - background scribble

$\mathrm{F}$ - flower scribble

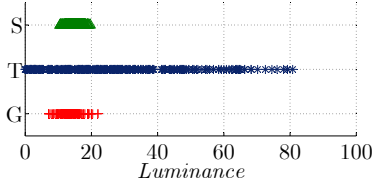

S - sky scribble

$\mathrm{T}$ - tree scribble

G - grass scribble

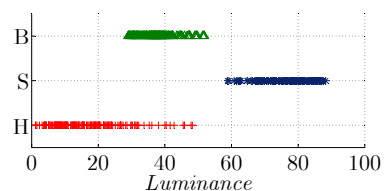

B - background scribble

S - skin scribble

$\mathrm{H}$ - hair scribble c)
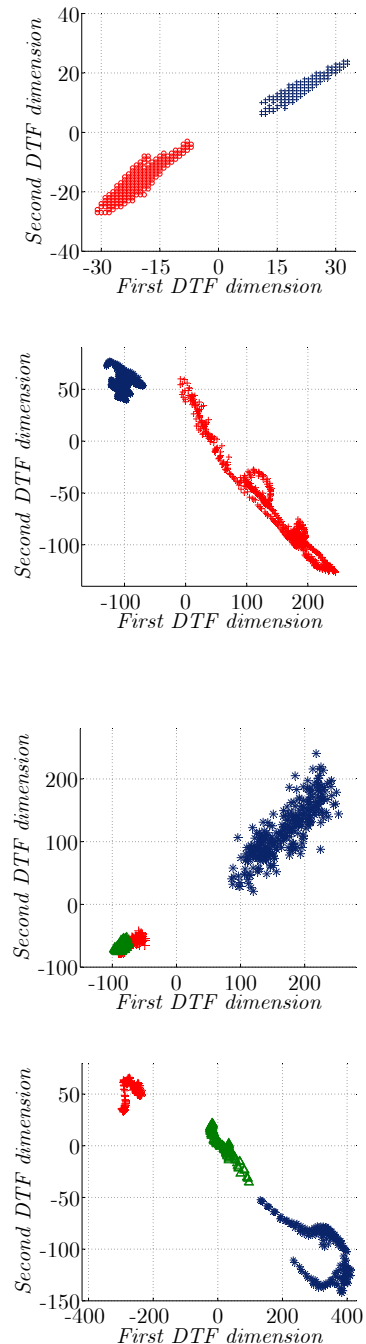

Figure 4 Scribble pixels (a) projected onto luminance (b) and 2D LDA (c) subspaces 
(G-red) scribbles in the tree image are overlapping each other even in the DTF subspace, but they both are well separated from the tree class. Although the achieved result is not perfect, it appeared sufficient to colorize the image properly as presented later in this section. For the last image, the three classes, i.e., "skin" (S-blue), "background" (B-green), and "hair" (H-red) are well separated.

For every scribble, a mean DTF feature vector is obtained and its DTF-distance $d_{D T F}(7)$ to every pixel in the image is computed in the DTF space. In this way, a DTF-distance map $d_{i}$ is obtained for every $i$ th scribble. Examples of the DTF-distance maps generated for two images are presented in Figure 5. Darker shade indicates smaller distance, i.e., greater similarity to the source scribble. It is clear from the Figure that the DTF-distance maps better differentiate between the scribbled regions than the original images themselves.

Potentially, the distance maps could be used directly for chrominance blending. In such a case, to obtain an $i$ th weight for a pixel $x$, the distance in DTF space $d_{i}(x)$ could be used instead of the total path cost $C_{i}(x)$ in (3). However, such approach does not beneft from pixels location and their geometrical distance from the scribbles. Also, continuity of the regions would not be guaranteed in this way. The DTF-distance maps can be used directly for some other applications, e.g., color transfer or video colorization, but here we found it better to treat them as a domain for color propagation. The local cost $\rho$ from pixel $x$ to $y$ equals the $y$ pixel value in the DTF-distance map $\left(\rho(x, y)=d_{i}(y)\right)$. For example, it can be concluded from Figure 5 (grass) that the upper-right sky region is texturally similar to the grass. This results from the overlapping in the DTF subspace observed earlier in Figure 4. Fortunately, these regions are located far from each other, which can be utilized using the propagation strategy. In this way these regions can be properly colorized, which would not be achieved using the distance maps directly for blending.

The propagation paths are determined so that they follow the texture similar to that covered by the source scribble. This is contrary to FIVC approach, with which the path is determined to minimize the luminance changes. An example of a difference between these two alternative approaches is given in Figure 6. It shows the propagation paths leading from a scribble to a selected pixel obtained using two methods. The paths determined using our method (b) do not leave the striped area, which makes it possible to colorize the image correctly (c). The paths obtained using a conventional method (d) show that the textural information is not taken into account during the propagation. This results in wrong colorization outcomes (e).

\subsection{Scribble boosting}

The method presented earlier in this section makes it possible to implement a complete colorization system; however, it has a serious drawback concerned with the precision. Although the regions having different texture are properly classified and separated in the DTF subspace, pixels lying at the region boundaries may be misclassified. The size of such misclassified areas depends on the kernel dimensions used for obtaining the basic textural features. This results in observing small halos at the region boundaries, which decreases the reality of the colorized images. Examples of these artifacts are presented in Figure 7.

If an image is densely annotated with scribbles, such effects are usually not observed using conventional methods. Following this observation, we decided to use

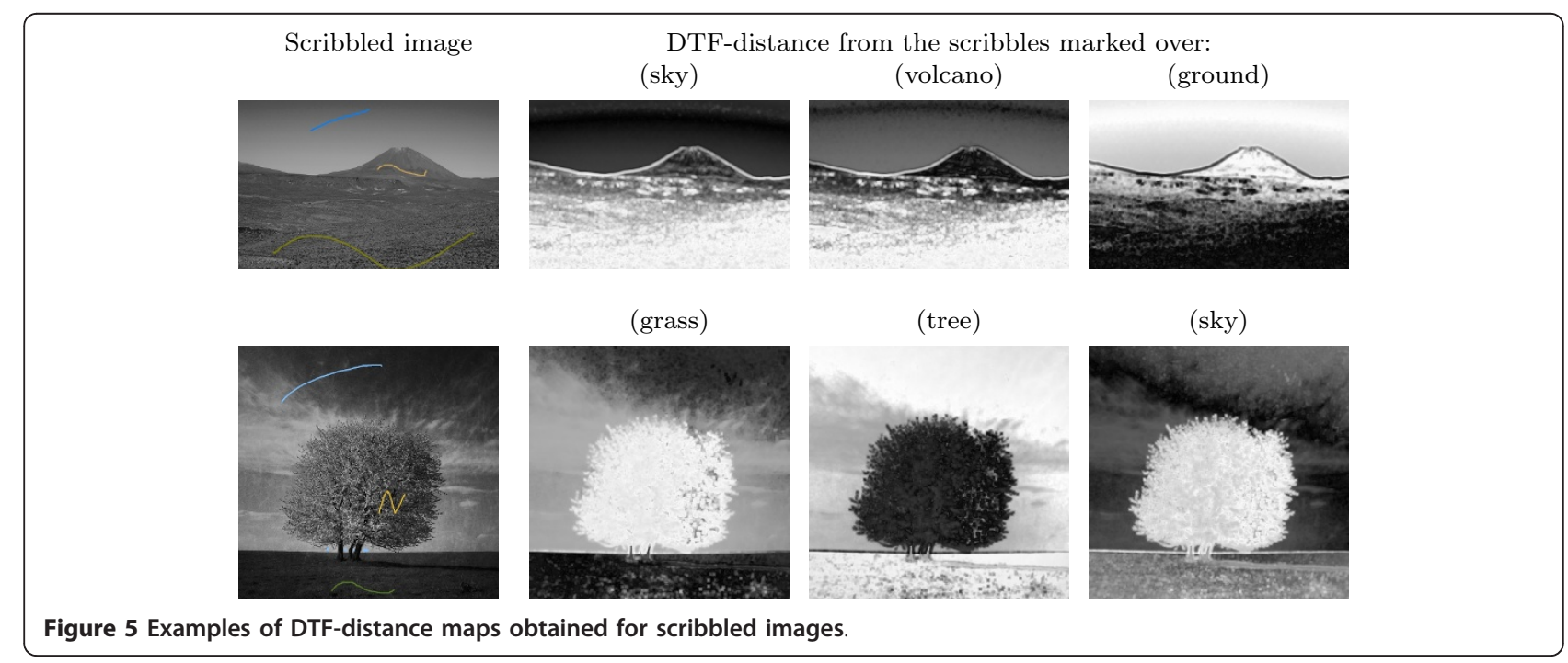


a)

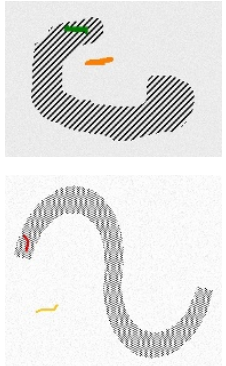

b)

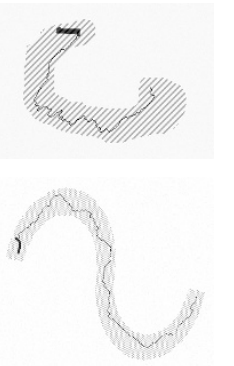

c)

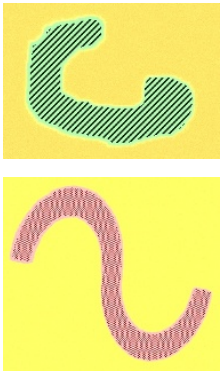

d)

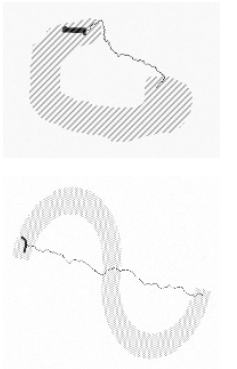

e)

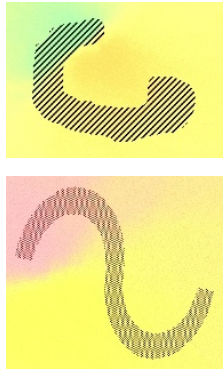

Figure 6 Scribbled images (a), propagation paths and colorized image obtained using our (b, c) and Yatziv's approach (d, e).

the DTF-based propagation to significantly enlarge (boost) the original scribbles, so that they cover the inner parts of the regions having similar texture without defining their boundaries. After that, the image with boosted scribbles is subject to the competitive propagation procedure presented in Section 4.

A flowchart of the proposed colorization method is given in Figure 8, and examples of resulting images obtained at subsequent steps of the procedure are demonstrated in Figure 9. The process consists of the following steps:

1. Basic textural features are extracted from every pixel in the original image as explained in Section 5.1.2. This operation creates an $M$-channel basic features image.

2. Each scribble forms an individual class of the basic feature vectors, extracted from the pixels covered by that scribble. This establishes a classified train set for LDA, which generates the projection matrix during training.

3. Based on the LDA projection matrix, the basic feature image is transformed into a DTF-features image.

4. A distance map in the DTF domain is obtained for every scribble as described in Section 5.2, using Equation (7).

5. Optimal paths from each scribble to every pixel in the image are determined using the DTF-distance maps. Here, we found it better to compute the total path cost as a maximal DTF-distance encountered on the path. Hence, the total path cost is obtained as $C_{\text {boost }}(p)=\max _{i=0 \ldots(l-1)}\{d(p(i))\}$. In this way the image is divided into mutually exclusive DTF regions, in which the individual scribbles win.

6. Every DTF region conquered by an individual scribble is shrunk using distance transform from the region's boundary. The shrinking margin size is determined based on an average length of the paths leading from the scribble to the boundary $\left(\bar{l}_{b}\right)$. During our experiments we set it to $0.750 .75 \bar{l}_{b}$, and we additionally provide that the original scribbles remain untouched after the shrinking. The shrunk regions are treated as the boosted scribbles for competitive propagation.

7. Competitive colorization is performed from the boosted scribbles (as outlined in Section 4). This operation generates the final colorized image.

Texture-based scribble boosting greatly facilitates the colorization of large image regions of uniform texture which are expected to obtain common chrominance. However, tiny image details are usually annotated with scribbles of specific colors which should not propagate far. Moreover, taking them into account for DTF computation may affect the discrimination power of the obtained feature space. Therefore, we allow the user to decide which scribbles are supposed to propagate only in their close neighborhood. We do not consider them for scribble boosting, and we also apply decreased scribble strength for them (e.g., $\sigma_{i}=0.1$ ). Although it may be
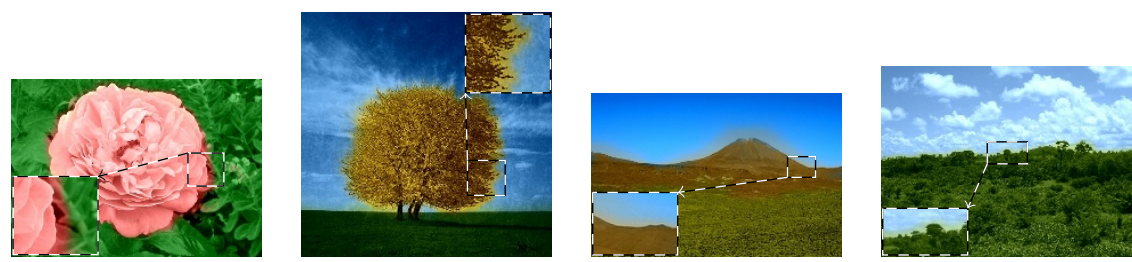

Figure 7 Examples of the halo efect observed for DTF-based colorization. 

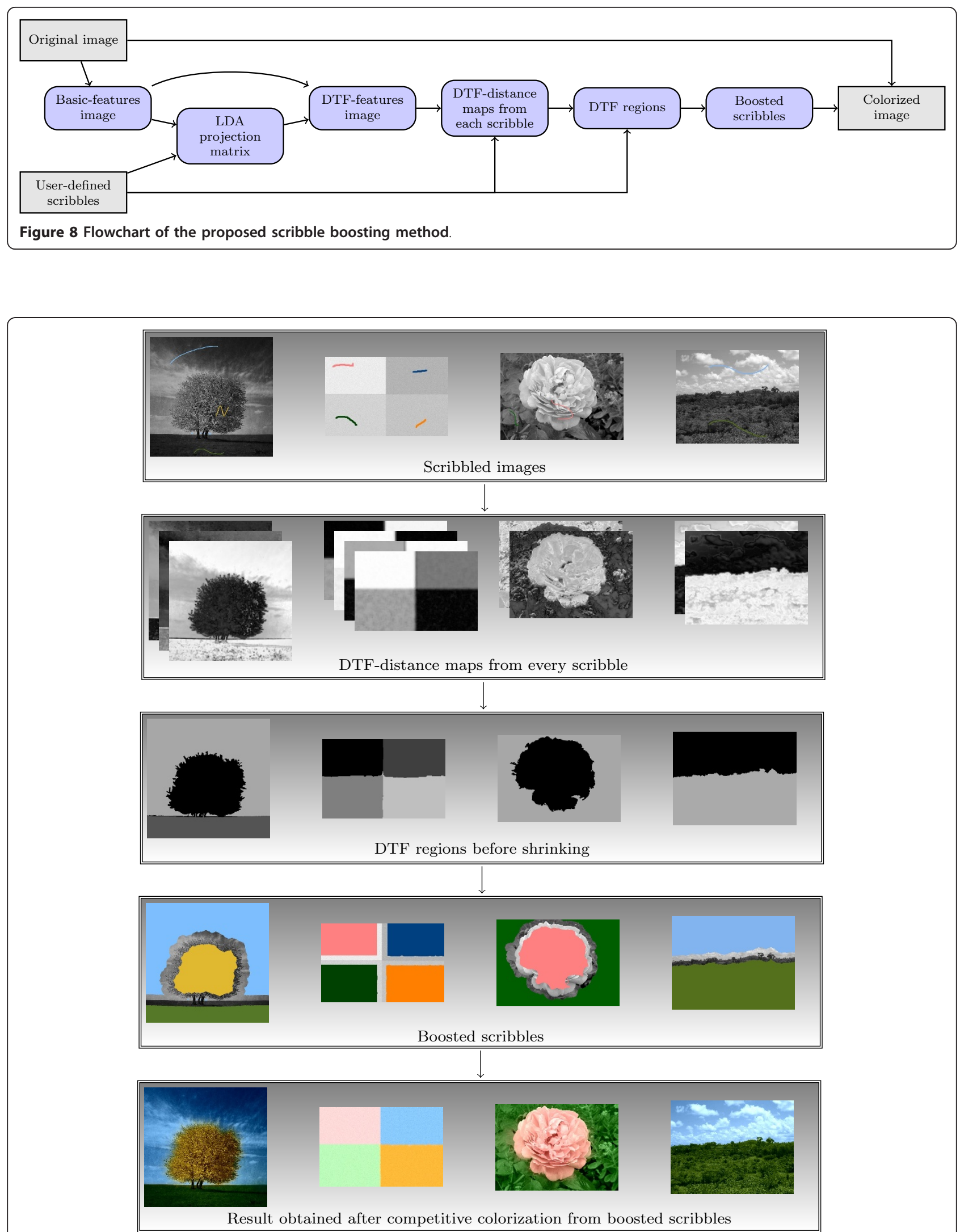

Figure 9 Examples of results obtained at selected steps of the colorization procedure. 
argued that this increases the user interaction, the overall gain attributed to the proposed technique is definitely beneficial.

\section{Experimental validation}

Experimental validation of the proposed colorization framework was focused on two main aspects. First, we investigated how sensitive the method is to amount and density of the scribbles. Then, we evaluated the obtained colorization result for a group of images, on the basis of mean opinion score (MOS). We compared the proposed method with two well-established colorization techniques: (1) Colorization using optimization (CUO) proposed by Levin [10] and (2) Fast image and video colorization (FIVC) proposed by Yatziv [3]. The first one is published in the form of MATLAB code and for the latter we used our implementation.

In Figures 10 and 11, we present two images colorized based on three scribble sets of different coverage (i.e., area covered by the scribbles expressed as percentage of the whole image area). The images were colorized using three methods, namely: (1) FIVC [3], (2) competitive image colorization [2], and (3) the proposed method. To provide fair comparison, we applied the blending weights to the FIVC method as well, as it is outlined in Section 3.2. The similarity measures between the obtained images are documented in Tables 1 and 2 . Here, the images colorized using the highest scribble coverage (9 and 5.8\%, accordingly) are compared with all the other images. We measured the similarity using
Peak Signal-to-Noise Ratio (PSNR), Structural Similarity Index (SSIM) [23], Normalized Color Distance (NCD) [24], and Universal Image Quality Index (UIQI) [25].

It may be observed that for large scribble coverage, three investigated methods deliver very similar outcome (top row in the Figures), and the differences are hardly visible, which is confirmed by the quantitative results. However, for smaller coverage, the images obtained using these methods differ significantly. It may be seen that both visually and quantitatively FIVC method is the most sensitive to the scribble coverage and it fails to colorize the images correctly for fewer scribbles. Competitive image colorization has higher stability, making it possible to colorize the image based on medium scribble coverage. However, it is only the proposed boosting technique which is very little dependent on the density and precision of the scribbles. Here, the colorized images are almost identical regardless of the scribble coverage, as it can be seen in the rightmost column in the Figures. Also, the similarity scores (highlighted in the tables) are very high between these images. It may be therefore concluded that using the proposed scribble boosting technique, it is possible to colorize images even from sparse scribble sets.

We have demonstrated that the scribble boosting does not depend much on the scribble coverage, making it possible to colorize images using small amount of scribbles. Furthermore, we have also investigated whether the location of scribbles may affect the colorization result. Figure 12 presents two images colorized from two

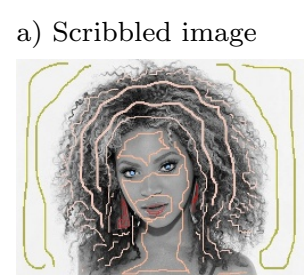

$9.0 \%$ coverage

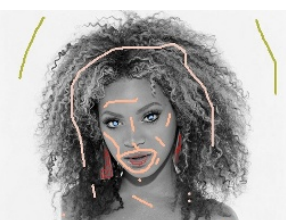

$3.0 \%$ coverage

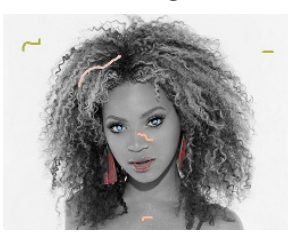

$0.7 \%$ coverage
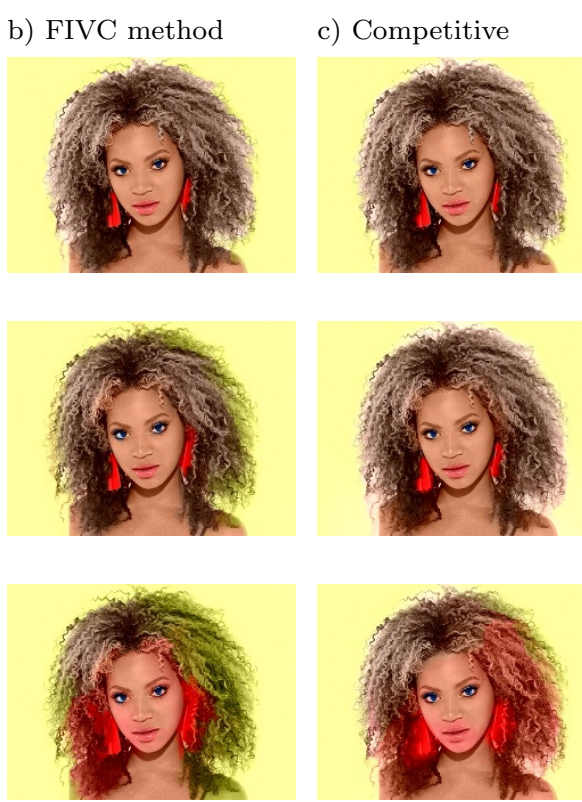
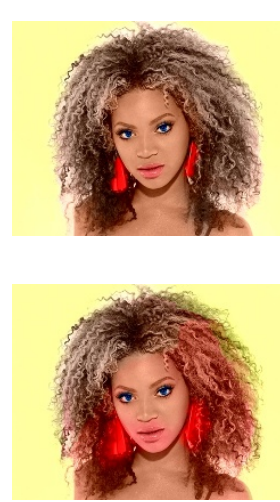
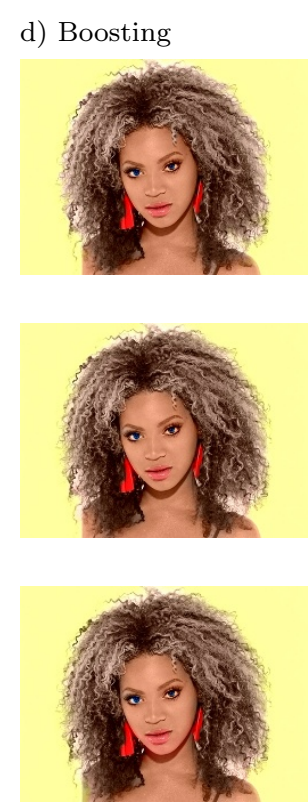

Figure 10 Facial image colorized using diferent methods based on three levels of scribble coverage. 

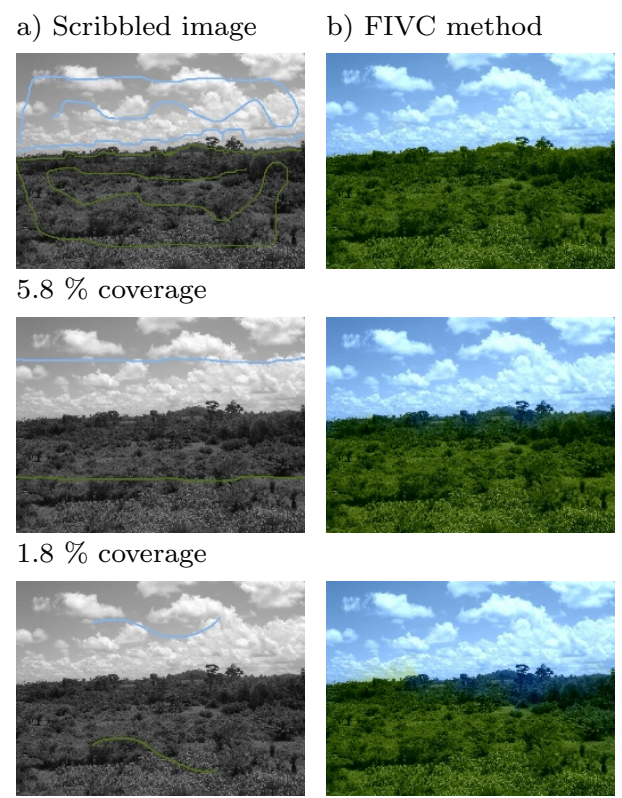

$0.8 \%$ coverage

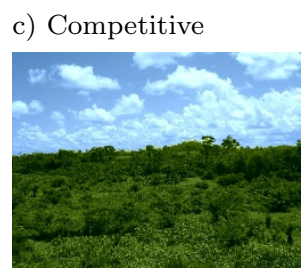

d) Boosting
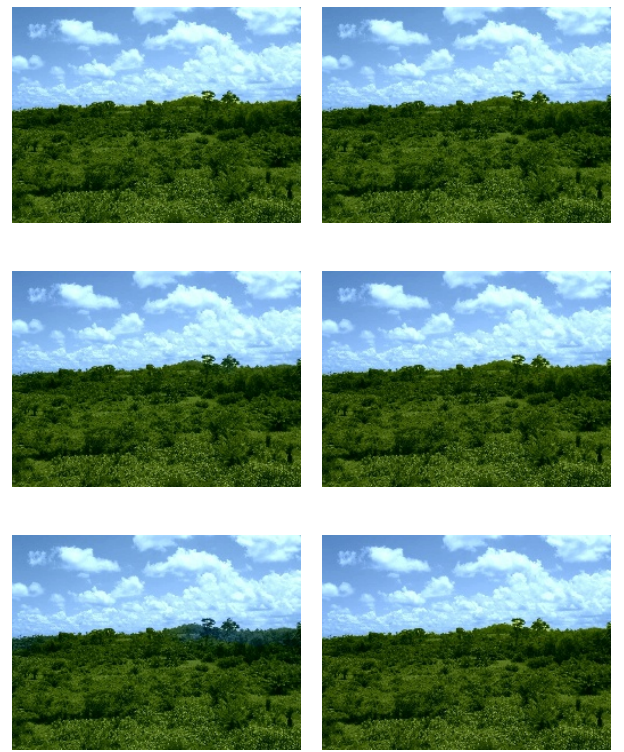

Figure 11 Landscape image colorized using diferent methods based on three levels of scribble coverage.

alternative sparse scribble sets. Although the scribbles supposed to colorize larger areas of uniform texture were positioned in different locations (upper row), it is hardly possible to spot any visual differences in the obtained results (bottom row). This is also indicated by high similarity scores presented in the Figure. Basically, it can be concluded that the scribble boosting is insensitive to changes in scribble location.

It is worth noting that the aim of image colorization is to achieve plausible visual impression on a human observer, and it is the visual effect which should determine how good an algorithm is. As it is difficult to measure visual attractiveness, we presented a group of 34 images to 38 observers. They were asked to rate the quality and naturalness of the images (scaled from 1 to 10). Thus, we obtained mean opinion scores (MOS) which are demonstrated in Table 3. Some examples of the images presented during this survey, as well as the scribbled grayscale images, are shown in Figure 13. All images used for the survey can be viewed at http://sun. aei.polsl.pl/ mkawulok/boosting/survey.pdf. The rest of images colorized using the proposed technique are shown in Figure 14. For the majority of cases the original color versions were available, and they were also

Table 1 Similarity scores between the images presented in Figure 10

\begin{tabular}{|c|c|c|c|c|c|c|c|c|c|c|}
\hline \multirow{2}{*}{\multicolumn{2}{|c|}{$\begin{array}{r}\downarrow \text { Method } \rightarrow \\
\text { Coverage } \rightarrow\end{array}$}} & \multicolumn{3}{|c|}{ FIVC } & \multicolumn{3}{|c|}{ Competitive } & \multicolumn{3}{|c|}{ Boosting } \\
\hline & & \multirow{2}{*}{$\begin{array}{c}9 \% \\
-\end{array}$} & \multirow{2}{*}{\begin{tabular}{c|}
$3 \%$ \\
27.29
\end{tabular}} & \multirow{2}{*}{$\begin{array}{l}\mathbf{0 . 7 \%} \\
21.42\end{array}$} & \multirow{2}{*}{$\begin{array}{c}9 \% \\
34.03\end{array}$} & \multirow{2}{*}{$\begin{array}{c}\mathbf{3 \%} \\
29.64\end{array}$} & \multirow{2}{*}{$\begin{array}{l}\mathbf{0 . 7 \%} \\
22.93\end{array}$} & \multirow{2}{*}{$\begin{array}{c}9 \% \\
34.02\end{array}$} & \multirow{2}{*}{$\begin{array}{c}3 \% \\
33.24\end{array}$} & \multirow{2}{*}{$\begin{array}{c}0.7 \% \\
32.5\end{array}$} \\
\hline FIVC (9\%) & PSNR & & & & & & & & & \\
\hline & SSIM & - & 0.973 & 0.905 & 0.994 & 0.982 & 0.930 & 0.994 & 0.992 & 0.991 \\
\hline & NCD & - & 0.067 & 0.207 & 0.024 & 0.053 & 0.164 & 0.026 & 0.032 & 0.036 \\
\hline & UIQI & - & 0.802 & 0.699 & 0.746 & 0.677 & 0.726 & 0.775 & 0.781 & 0.741 \\
\hline \multirow[t]{4}{*}{ Comp. (9\%) } & PSNR & 34.03 & 26.41 & 21.29 & - & 31.19 & 23.04 & 37.27 & 34.22 & 33.06 \\
\hline & SSIM & 0.994 & 0.969 & 0.903 & - & 0.987 & 0.931 & 0.997 & 0.994 & 0.992 \\
\hline & NCD & 0.024 & 0.075 & 0.21 & - & 0.46 & 0.16 & 0.018 & 0.03 & 0.034 \\
\hline & UIQI & 0.746 & 0.765 & 0.709 & - & 0.569 & 0.687 & 0.795 & 0.781 & 0.78 \\
\hline \multirow[t]{4}{*}{ Boost. (9\%) } & PSNR & 34.02 & 26.53 & 21.2 & 37.27 & 30.63 & 22.89 & - & 37.46 & 35.49 \\
\hline & SSIM & 0.994 & 0.97 & 0.901 & 0.997 & 0.985 & 0.93 & - & 0.997 & 0.995 \\
\hline & NCD & 0.026 & 0.075 & 0.212 & 0.018 & 0.051 & 0.164 & - & 0.017 & 0.021 \\
\hline & UIQI & 0.775 & 0.877 & 0.822 & 0.795 & 0.704 & 0.691 & - & 0.935 & 0.947 \\
\hline
\end{tabular}

Bold values indicate the best score. 
Table 2 Similarity scores between the images presented in Figure 11

\begin{tabular}{|c|c|c|c|c|c|c|c|c|c|c|}
\hline \multirow{2}{*}{\multicolumn{2}{|c|}{$\begin{array}{l}\downarrow \text { Method } \rightarrow \\
\quad \text { Coverage } \rightarrow\end{array}$}} & \multirow{3}{*}{$\begin{array}{c}5.8 \% \\
-\end{array}$} & \multicolumn{2}{|c|}{ FIVC } & \multicolumn{3}{|c|}{ Competitive } & \multicolumn{3}{|c|}{ Boosting } \\
\hline & & & \multirow{2}{*}{$\begin{array}{l}1.8 \% \\
27.55\end{array}$} & \multirow{2}{*}{$\begin{array}{l}\mathbf{0 . 8 \%} \\
25.07\end{array}$} & \multirow{2}{*}{$\begin{array}{l}5.8 \% \\
30.26\end{array}$} & \multirow{2}{*}{$\begin{array}{l}1.8 \% \\
30.46\end{array}$} & \multirow{2}{*}{$\begin{array}{l}0.8 \% \\
28.45\end{array}$} & \multirow{2}{*}{\begin{tabular}{r|}
$5.8 \%$ \\
37.6
\end{tabular}} & \multirow{2}{*}{$\begin{array}{l}1.8 \% \\
36.51\end{array}$} & \multirow{2}{*}{$\begin{array}{l}\mathbf{0 . 8 \%} \\
36.51\end{array}$} \\
\hline FIVC (5.8\%) & PSNR & & & & & & & & & \\
\hline & SSIM & - & 0.942 & 0.919 & 0.966 & 0.963 & 0.949 & 0.987 & 0.985 & 0.985 \\
\hline & NCD & - & 0.069 & 0.102 & 0.03 & 0.035 & 0.054 & 0.013 & 0.022 & 0.022 \\
\hline & UIQI & - & 0.891 & 0.874 & 0.92 & 0.917 & 0.901 & 0.937 & 0.938 & 0.937 \\
\hline \multirow[t]{4}{*}{ Comp. (5.8\%) } & PSNR & 30.26 & 28.86 & 25.73 & - & 37.45 & 31.01 & 30.45 & 30.78 & 30.79 \\
\hline & SSIM & 0.966 & 0.965 & 0.941 & - & 0.992 & 0.975 & 0.975 & 0.976 & 0.976 \\
\hline & NCD & 0.03 & 0.053 & 0.086 & - & 0.014 & 0.034 & 0.022 & 0.03 & 0.03 \\
\hline & UIQI & 0.92 & 0.927 & 0.9 & - & 0.961 & 0.941 & 0.954 & 0.953 & 0.953 \\
\hline \multirow[t]{4}{*}{ Boost. (5.8\%) } & PSNR & 37.6 & 27.12 & 24.74 & 30.45 & 30.81 & 28.41 & - & 40.00 & 39.97 \\
\hline & SSIM & 0.987 & 0.944 & 0.919 & 0.975 & 0.971 & 0.954 & - & 0.996 & 0.996 \\
\hline & NCD & 0.013 & 0.068 & 0.101 & 0.022 & 0.028 & 0.049 & - & 0.015 & 0.015 \\
\hline & UIQI & 0.937 & 0.91 & 0.885 & 0.954 & 0.948 & 0.926 & - & 0.975 & 0.975 \\
\hline
\end{tabular}

Bold values indicate the best score.

presented to the observers in order to establish the reference level. It may be noticed that the colorized images differ much from the originals. This is because the scribbles do not indicate all the details, and in some cases the chrominance assigned to a scribble is different from the chrominance in the original image. Moreover, the chrominance variance is much higher in the originals, while the colorized images inherit only a mixture of the chrominance values assigned to the scribbles. This disadvantage may be overcome by assigning a color palette to every scribble instead of a single chrominance, analogously to the luminance keying [4]. Despite of that, the colorized images scores were quite close to those obtained by the originals, and even higher in some cases. This was mainly due to the fact that the area conquered by the individual scribbles appears correct and natural using the proposed scribble boosting. Although these rates are lower than for the originals, they are definitely better than those obtained for the alternative conventional techniques.

\section{Conclusions and future work}

This paper presents a new method for image colorization which utilizes local textural features. We have demonstrated that texture is a powerful source of information that supports the colorization process. The proposed scribble boosting technique increases the original scribbles on the basis of discriminant textural features. This facilitates interactive image colorization, and decreases the required density and precision of the scribbles. DTF are determined for every individual image using linear discriminant analysis, which makes the method adaptive to local conditions.

The method can be further extended and used for color transfer and video colorization. Once the DTF space is created for a given image, it may be applied to

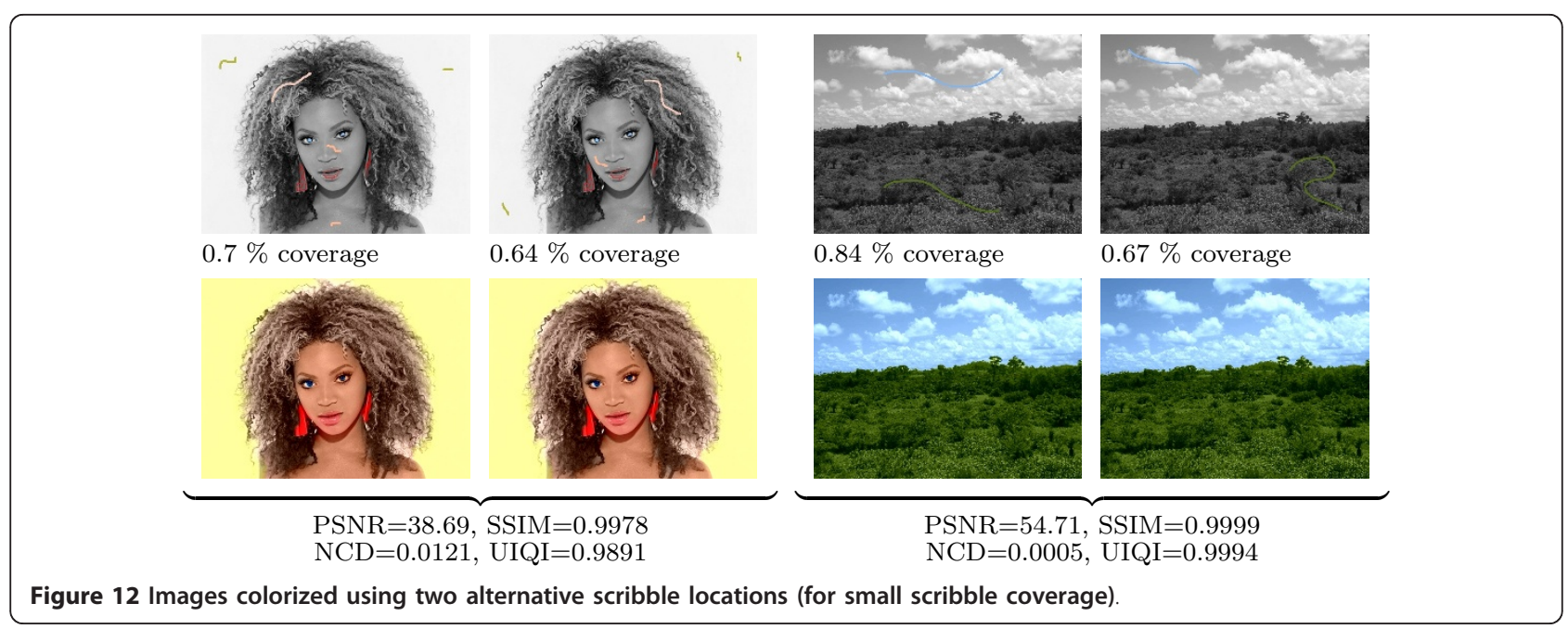


Table 3 Mean opinion scores obtained for original and colorized images

\begin{tabular}{lcccc}
\hline & $\begin{array}{c}\text { Original } \\
\text { image }\end{array}$ & $\begin{array}{c}\text { FIVC } \\
\text { method }\end{array}$ & $\begin{array}{c}\text { CUO } \\
\text { method }\end{array}$ & $\begin{array}{c}\text { Scribble } \\
\text { boosting }\end{array}$ \\
\hline Elephant & $8.91 \pm 1.26$ & $3.39 \pm 2.12$ & $3.26 \pm 1.78$ & $7.97 \pm 1.29$ \\
Face A & $7.76 \pm 1.78$ & $4.91 \pm 1.75$ & $4.38 \pm 1.99$ & $7.53 \pm 1.73$ \\
Face B & $8.44 \pm 1.85$ & $2.03 \pm 1.17$ & $2.24 \pm 1.65$ & $4.15 \pm 2.05$ \\
Face C & $5.82 \pm 2.26$ & $4.09 \pm 2.17$ & $4.24 \pm 1.99$ & $7.41 \pm 1.99$ \\
Face D & - & $5.47 \pm 2.03$ & $6.18 \pm 2.11$ & $6.97 \pm 1.93$ \\
Flower & $8.91 \pm 1.56$ & $4.21 \pm 2.12$ & $3.71 \pm 1.99$ & $8.03 \pm 1.88$ \\
Forest & $8.56 \pm 1.88$ & $4.97 \pm 1.88$ & $3.88 \pm 2.23$ & $7.44 \pm 1.62$ \\
Meadow & $8.24 \pm 1.72$ & $4.71 \pm 1.99$ & $5.74 \pm 1.96$ & $6.94 \pm 1.92$ \\
Tree & - & $3.06 \pm 1.52$ & $3.82 \pm 1.66$ & $4.82 \pm 1.95$ \\
\hline Average & $8.09 \pm 1.08$ & $4.09 \pm 1.09$ & $4.16 \pm 1.2$ & $6.81 \pm 1.38$ \\
\hline
\end{tabular}

any other image having similar contents. This extension will be explored during our future works.

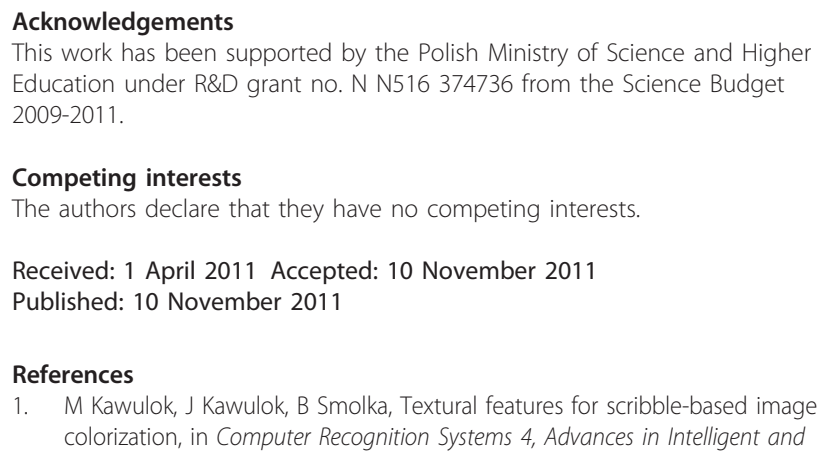

Competing interests

The authors declare that they have no competing interests.

Received: 1 April 2011 Accepted: 10 November 2011

Published: 10 November 2011

\section{References}

1. M Kawulok, J Kawulok, B Smolka, Textural features for scribble-based image colorization, in Computer Recognition Systems 4, Advances in Intelligent and

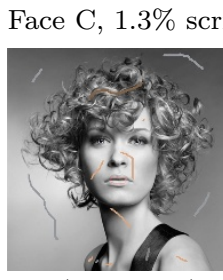

(Scribbled)

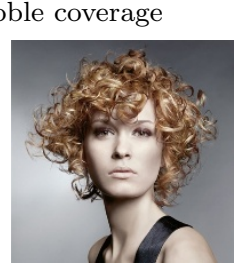

(Original)

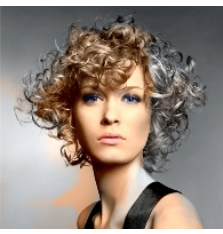

(FIVC)

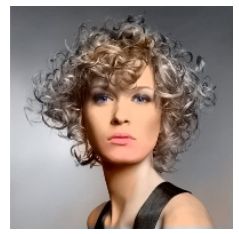

(CUO)

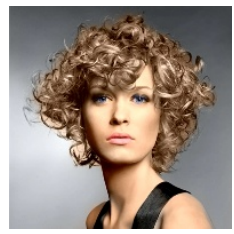

(Boosting)

Meadow, $0.4 \%$ scribble coverage

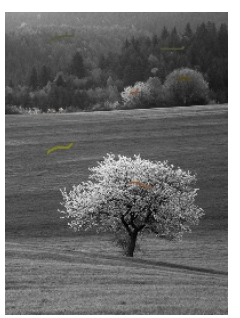

(Scribbled)

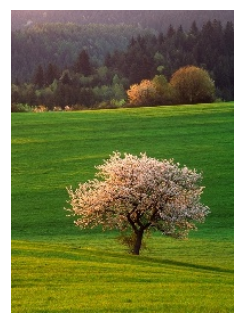

(Original)

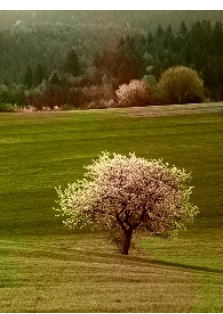

(FIVC)

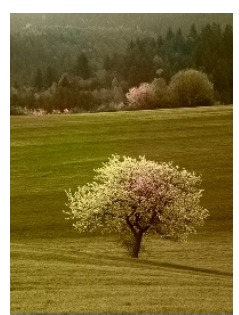

$(\mathrm{CUO})$

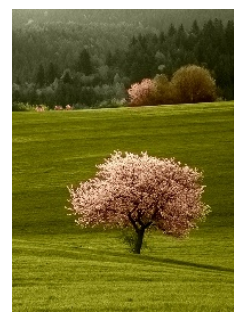

(Boosting)

Elephant, $0.8 \%$ scribble coverage

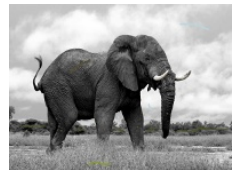

(Scribbled)

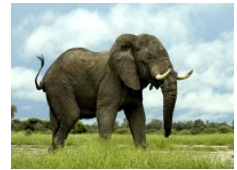

(Original)

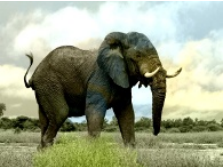

(FIVC)

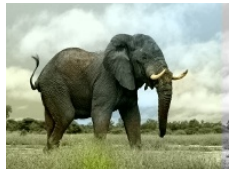

(CUO)

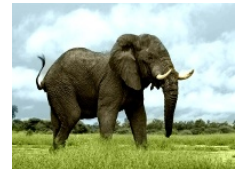

(Boosting)

Figure 13 Selected examples of colorized images used in the survey

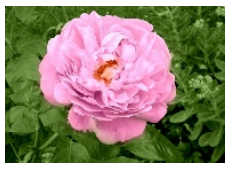

Flower

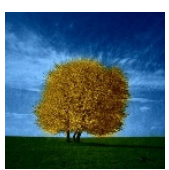

Tree

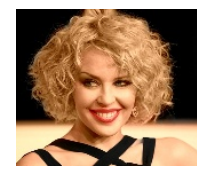

Face A

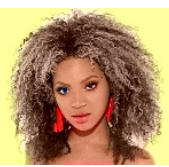

Face B

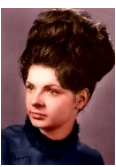

Face D

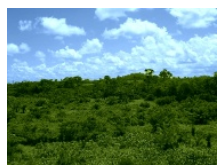

Forest

Figure 14 Examples of images used in the survey, colorized using scribble boosting 
Soft Computing, vol. 95, ed. by R Burduk, M Kurzynski, M Wozniak, and A Zolnierek (Springer, Berlin, 2011), pp. 269-278

2. M Kawulok, B Smolka, Competitive image colorization, in IEEE ICIP, IEEE, Hong Kong, pp. 405-408 (2010)

3. L Yatziv, G Sapiro, Fast image and video colorization using chrominance blending. IEEE Trans Image Proc. 15(5), 1120-1129 (2006)

4. R Gonzalez, R Woods, Digital Image Processing, (Addison Wesley Publishing, Boston, 1987)

5. T Welsh, M Ashikhmin, K Mueller, Transferring color to greyscale images. ACM Trans Graph (TOG). 21(3), 277-280 (2002)

6. U Lipowezky, Grayscale aerial and space image colorization using texture classifcation. Pattern Rec Lett. 27(4), 275-286 (2006). doi:10.1016/j. patrec.2005.08.009

7. D Sykora, J Burianek, J Zara, Unsupervised colorization of black-and-white cartoons, in SIGGRAPH, ACM, Los Angeles, California, pp. 121-127 (2004)

8. T Horiuchi, Colorization algorithm using probabilistic relaxation. Image Vis Comput. 22(3), 197-202 (2004). doi:10.1016/j.imavis.2003.08.004

9. T Horiuchi, H Kotera, Colorization for monochrome image with texture, in Proceedings of 13th Color Imaging Conference, IS\&T, Scottsdale, Arizona, pp. 245-250 (2005)

10. A Levin, D Lischinski, Y Weiss, Colorization using optimization, in SIGGRAPH, ACM, Los Angeles, California, pp. 689-694 (2004)

11. P Lagodzinski, B Smolka, Digital image colorization based on probabilistic distance transform, in ELMAR, 2008, vol. 2. (IEEE, Zadar, Croatia, 2008), pp. 495-498

12. V Konushin, $V$ Vezhnevets, Interactive image colorization and recoloring based on coupled map lattices, GraphiCon, (GraphiCon, Novosibirsk Akademgorodok, Russia, 2006), pp. 231-234

13. T Kim, K Lee, S Lee, Edge-preserving colorization using data-driven random walks with restart, IEEE ICIP, (IEEE, Cairo, Egypt, 2009), pp. 1661-1664

14. L Ikonen, P Toivanen, Distance and nearest neighbor transforms on graylevel surfaces. Pattern Rec. Lett. 28(5), 604-612 (2007). doi:10.1016/j. patrec.2006.10.010

15. E Mortensen, W Barrett, Interactive segmentation with intelligent scissors. Graph Models Image Proc. 60(5), 349-384 (1998). doi:10.1006/ gmip.1998.0480

16. J Heu, D Hyun, C Kim, S Lee, Image and video colorization based on prioritized source propagation, in IEEE ICIP, IEEE, Cairo, Egypt, pp. 465-468 (2009)

17. J Zhang, S Lazebnik, C Schmid, Local features and kernels for classifcation of texture and object categories: a comprehensive study. Int J Comput Vis. 73, 213-238 (2007). doi:10.1007/s11263-006-9794-4

18. RM Haralick, Statistical and structural approaches to texture. Proc IEEE. 67(5), 786-804 (1979)

19. T Ojala, M Pietikäinen, T Mäenpää, Multiresolution gray-scale and rotation invariant texture classifcation with local binary patterns. IEEE Trans Pattern Anal Mach Intell. 24(7), 971-987 (2002). doi:10.1109/TPAMI.2002.1017623

20. J Portilla, E Simoncelli, A parametric texture model based on joint statistics of complex wavelet coefficients. Int J Comput Vis. 40(1), 49-71 (2000). doi:10.1023/A:1026553619983

21. M Varma, A Zisserman, A statistical approach to texture classifcation from single images. Int J Comput Vis. 62, 61-81 (2005)

22. G Seber, Multivariate Observations, (Wiley, New York, 1984)

23. Z Wang, A Bovik, HR Sheikh, EP Simoncelli, Image quality assessment: from error visibility to structural similarity. IEEE Trans Image Process. 13(4), 600-612 (2004). doi:10.1109/TIP.2003.819861

24. R Lukac, K Plataniotis, D Hatzinakos, M Aleksic, A novel cost efective demosaicing approach. IEEE Trans Consum Electron. 50(1), 256-261 (2004). doi:10.1109/TCE.2004.1277871

25. Z Wang, A Bovik, A universal image quality index. IEEE Signal Process Lett. 9(3), 81-84 (2002). doi:10.1109/97.995823

doi:10.1186/1687-6180-2011-99

Cite this article as: Kawulok and Smolka: Texture-adaptive image

colorization framework. EURASIP Journal on Advances in Signal Processing $20112011: 99$.

\section{Submit your manuscript to a SpringerOpen ${ }^{\mathcal{O}}$ journal and benefit from:}

- Convenient online submission

- Rigorous peer review

- Immediate publication on acceptance

- Open access: articles freely available online

- High visibility within the field

- Retaining the copyright to your article

Submit your next manuscript at $\gg$ springeropen.com 